\title{
ATCA radio observations of compact planetary nabulae
}

\author{
Marcin Hajduk ${ }^{1}$ and Albert A. Zijlstra ${ }^{2}$ \\ ${ }^{1}$ Nicolaus Copernicus Astronomical Center, ul. Rabiańska 8, 87-100 Toruń, Poland \\ email: Marcin.Hajduk@ncac.torun.pl \\ ${ }^{2}$ Jodrell Bank Centre for Astrophysics, Alan Turing Building, Manchester M13 9PL, UK \\ email: a.zijltra@manchester.ac.uk
}

\begin{abstract}
We present new observations in the radio continuum of 31 planetary nebulae at 5 and $8 \mathrm{GHz}$ with the Australian Telescope Compact Array. The observations are used to invesigate properties of the interstellar extinction toward Galactic Bulge.
\end{abstract}

Keywords. planetary nebulae: general, dust, extinction, Galaxy: bulge

\section{Introduction}

Radio continuum emission brings information on much needed parameters of Planetary Nebulae (PNe): their extents, emission measure or density gradient. The radio emission is not affected by the interstellar extinction. In combination with the optical flux it may reveal the properties of the interstellar extinction.

Stasińska et al. (1992) and Tylenda et al. (1992) have compared the Balmer decrement flux ratios and $5 \mathrm{GHz}$ flux to the $\mathrm{H} \beta$ flux ratios for the Galactic Bulge PNe. They suggested that the ratio of the total to selective absorption $R_{V}$ value differs from 3.1, typical for the Galactic disk. More recently, Ruffle et al. (2004) has estimated an average value of $R_{V}$ to be equal to 2.0 for the Galactic Bulge PNe. Here, we re-examine their results with a new radio data set from the ATCA (Australian Telescope Compact Array) interferometer.

\section{Determination of the $R_{V}$}

The radio data were collected with the ATCA on November 16, 17 and 18, 2003 at 5 and $8 \mathrm{GHz} .5 \mathrm{~min}$ integrations of PNe were altered with observations of phase calibrators in the 'snap-shot' mode. Each nebula was observed approximately one hour in total. 31 PNe were observed, 30 of them being likely members of the Galactic bulge.

16 out of the 30 objects had already been observed with the VLA at $5 \mathrm{GHz}$. The fluxes measured with the ATCA exceed the VLA fluxes by 10-15\% (median is 15\%) at $5 \mathrm{GHz}$ (Figure 1). The discrepancy is higher for PNe fainter than $10 \mathrm{mJy}$. An extreme case is PN G004.8+02.0, with the ATCA flux 3.5 times higher than the VLA flux. The uncertainty of the flux scale may account for only $7 \%$ difference.

The observed ratio $S_{5 \mathrm{GHz}} / S_{8 \mathrm{GHz}}$ varies between $\sim 1-1.25$. The $5 \mathrm{GHz}$ flux of an optically thin PN should exceed the $8 \mathrm{GHz}$ flux by 7 percent. Both higher than expected $S_{5 \mathrm{GHz}} / S_{8 \mathrm{GHz}}$ flux ratio and the discrepancy between the VLA and ATCA fluxes may be explained by slightly underestimated $5 \mathrm{GHz}$ flux of the ATCA calibrator.

$R_{V}$ values calculated for the nebulae meeting the selection criteria $\left(100>S_{5 \mathrm{GHz}}>10\right.$, $\left.T_{\mathrm{b}}<1000 K\right)$ are presented in the Figure 2 . The obtained values of $R_{V}$ range from 0.84 to 2.85 for the Bulge nebulae. The mean $R_{V}$ for 20 planetary nebulae equals to 2.0. Our 


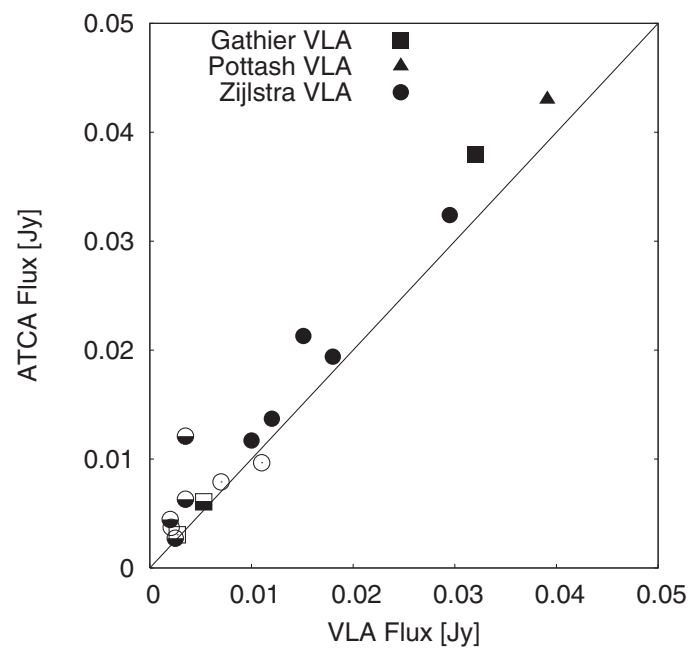

Figure 1. Comparison of the ATCA and the VLA fluxes.

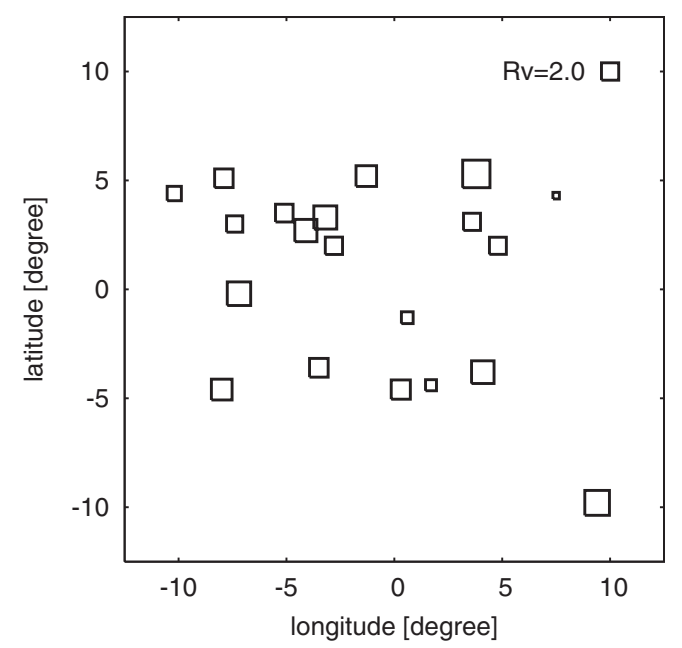

Figure 2. $R_{V}$ parameter for PNe determined from the ATCA observations combined with the values given by Ruffle et al. (2004).

results confirm these obtained by Ruffle (2004). The sample of the objects has increased from 13 up to 20 .

\section{Acknowledgements}

This work was financially supported by MNiSW of Poland through grant No. N N203511838.

\section{References}

Ruffle, P., Zijlstra, A. A., Walsh, J. R., Gray, M. D., Gesicki, K., Minniti, D., \& Comeron, F. 2004, MNRAS, 353, 796

Stasińska, G., Tylenda, R., Acker, A., \& Stenholm, B. 1992, A\&A, 266, 486

Tylenda, R., Acker, A., Stenholm, B., \& Koeppen, J. 1992, AAS, 95, 337

Zijlstra, A. A., Pottash, S. R., \& Bignell, C. 1989, AAS, 79, 329

van Hoof, P. A. M. 2000, MNRAS, 314, 99 